\title{
El estrés crónico como mediador de la relación entre la posición socioeconómica y el cumplimiento del tratamiento farmacológico de pacientes hipertensos
}

\author{
Deivis Nicolás Guzmán-Tordecilla ${ }^{1}$, Andrés Ignacio Vecino-Ortiz²,3, Diego Lucumí1, \\ Graciela Mentz ${ }^{4}$ \\ ${ }^{1}$ Escuela de Gobierno, Universidad de los Andes, Bogotá, D.C., Colombia \\ 2 Instituto de Salud Pública, Pontificia Universidad Javeriana, Bogotá, D.C., Colombia \\ ${ }^{3}$ Department of International Health, Johns Hopkins Bloomberg School of Public Health, \\ Baltimore, MD, USA \\ ${ }^{4}$ Department of Health Behavior and Health Education, University of Michigan, Ann Arbor, MI, USA
}

Introducción. La hipertensión arterial sistémica es un problema de salud pública en el mundo. En Colombia, su prevalencia es del $25 \%$ y la mortalidad es alta. Los factores psicosociales que afectan el cumplimiento del tratamiento farmacológico no han sido estudiados suficientemente. En otros países, se ha estudiado el papel del estrés crónico en la relación entre la posición socioeconómica y el cumplimiento del tratamiento farmacológico antihipertensivo.

Objetivo. Examinar el papel del estrés crónico como mediador de la relación entre la posición socioeconómica y el cumplimiento del tratamiento farmacológico, en pacientes hipertensos de 45 a 70 años en el 2015 y el 2016.

Materiales y métodos. Se hizo un estudio transversal de una población de pacientes hipertensos. Los datos provienen de la muestra seleccionada para el programa "De todo corazón" en Bogotá, Medellín y Quibdó. El análisis estadístico de los datos se hizo mediante análisis factorial y regresiones multivariadas.

Resultados. Los resultados confirmaron una asociación positiva entre la posición socioeconómica y el grado de cumplimiento del tratamiento farmacológico, y una relación negativa entre la primera y el estrés crónico. Además, se evidenció que el estrés tiene una asociación negativa con el grado de cumplimiento.

Recibido: 19/10/2018

Aceptado: $15 / 08 / 2019$

Publicado: $27 / 08 / 2019$

\section{Citación:}

Guzmán-Tordecilla DN, Vecino-Ortiz Al, Lucumí $D$, Mentz G. El estrés crónico como mediador de la relación entre la posición socioeconómica y el cumplimiento del tratamiento farmacológico en pacientes hipertensos. Biomédica. 2020;40:243-56. https://doi.org/10.7705/biomedica.4780

\section{Correspondencia:}

Deivis Nicolás Guzmán-Tordecilla, Carrera 5 No 1826, piso 301, Bogotá, D.C., Colombia

Teléfono: (51) 3394949

deivy-gt@hotmail.com

Contribución de los autores:

Todos los autores participaron en el diseño metodológico, el análisis de los datos, la redacción y la revisión final del manuscrito.

Financiación:

Este estudio fue financiado por el Grupo de Investigación de Factores Psicosociales en Psicología Clínica y de la Salud de la Universidad de los Andes en el marco del Programa "Determinantes sociales e inequidades en el control de la hipertensión arterial: mecanismos, impacto e intervención," el cual recibe recursos de la Vicerrectoría de Investigaciones de la Universidad de los Andes.

Conflicto de intereses:

Los autores declaran no haber tenido conflictos de intereses de ningún tipo durante el desarrollo del presente estudio.
Conclusiones. Los resultados sugieren que el estrés no es un mediador entre la posición socioeconómica y el cumplimiento del tratamiento farmacológico antihipertensivo en Colombia. Se requieren estudios adicionales para confirmar estas relaciones con una muestra más amplia.

Palabras clave: estrés psicológico; hipertensión; cumplimiento del tratamiento; cumplimiento de la medicación; clase social; Colombia.

Chronic stress as a mediator of the relationship between socioeconomic status and pharmacological adherence in hypertensive patients

Introduction: High blood pressure is a public health problem worldwide. In Colombia, its prevalence is $25 \%$ with a high mortality rate. The psychosocial factors affecting pharmacological adherence among patients have not been sufficiently studied and despite international evidence on their impact, in Colombia, there is a paucity of research on the role of chronic stress in the relationship between socioeconomic status and pharmacological adherence.

Objective: To examine the role of chronic stress in the relationship between socioeconomic status and pharmacological adherence in hypertensive patients aged 45 to 70 years old in three Colombian cities between 2015 and 2016.

Materials and methods: We conducted a cross-sectional study in a population of hypertensive patients. Data for this study came from the first wave of longitudinal study aimed at examining social factors associated with the control of hypertension in Bogotá, Medellín, and Quibdó. Patients with hypertension were selected randomly from a sample of those participating in the hypertension control program De todo corazón. For the statistical analysis of the data, we used factorial analysis and multivariate regressions.

Results: We found a positive association between socioeconomic status and the degree of pharmacological adherence and a negative one with chronic stress. Besides, evidence was found that stress has a negative association with the degree of adherence.

Conclusions: The results suggest that stress is not a likely mediator between socioeconomic status and the pharmacological adherence of hypertensive patients in Colombia. Additional studies are required to confirm these relationships with a larger sample. 
Keywords: Stress, psychological; hypertension; treatment adherence and compliance; medication adherence; social class; Colombia.

La hipertensión arterial sistémica se considera un problema de salud pública a nivel mundial $(1,2)$. Se estima que su prevalencia es mayor en Latinoamérica $(39,1 \%)$ que en otros partes del mundo (32,3\%) (3). Del total de personas hipertensas en el mundo, 9,4 millones mueren cada año, lo que convierte esta afección en el principal factor de riesgo de la enfermedad cardiovascular, con el $31 \%$ (17,5 millones) del total de las muertes reportadas atribuibles a la misma $(4,5)$. Además, se prevé que, en el 2025, habrá 1.500 millones de hipertensos en todo el mundo (2).

También en Colombia, la hipertensión arterial sistémica es un problema de salud pública. La prevalencia estimada a partir de una muestra poblacional de adultos fue de $25 \%$ (6) y, en el 2015, se informó que había sido la causa básica de muerte de 5.157 personas (7). Esta cifra podría ser mayor, ya que se ha establecido que la hipertensión arterial es responsable, aproximadamente, del $45 \%$ de las muertes por cardiopatías y del $51 \%$ de aquellas por accidente cerebrovascular (4).

Se ha encontrado que, en general, las personas con hipertensión arterial sistémica poco cumplen con el tratamiento farmacológico $(1,8-10)$, lo que se traduce en su control deficiente, y en disminución de la calidad de vida y de la supervivencia de quienes la padecen.

Según Gasperin, et al., los pacientes que logran acceder al tratamiento farmacológico tienen un alto riesgo de abandonarlo a largo plazo, debido a múltiples factores psicosociales y a la naturaleza asintomática de la enfermedad (1), lo que explicaría el poco control (30\%) de la hipertensión arterial en el mundo (11). Se ha establecido, asimismo, que las personas que más incumplen el tratamiento y menos controlan su presión arterial son las que tienen una posición socioeconómica más baja $(11,12)$.

La evidencia acumulada ha demostrado que la posición socioeconómica está asociada con los resultados en salud (13-15). Grotto, et al., explican que esta puede influir en los comportamientos en salud y en el acceso a los servicios de salud o los medicamentos antihipertensivos (13). Además, constituye un factor que expone a las personas a condiciones de gran demanda psicológica y de estrés crónico (13-18). En Colombia, una significativa proporción de la población muestra condiciones socioeconómicas como bajo nivel educativo, elevada informalidad laboral y bajos ingresos (19-20), todo lo cual podría contribuir a exponerla al estrés crónico. Diversos autores han encontrado que la hipertensión arterial sistémica está asociada con el estrés (1,21-23). La exposición al estrés crónico no solo tiene efectos fisiológicos, sino que también genera comportamientos de riesgo para la salud, entre los que se encuentra el incumplimiento del tratamiento antihipertensivo $(24,25)$.

El efecto del estrés crónico en el cumplimiento del tratamiento antihipertensivo farmacológico puede explicarse parcialmente por la carga cognitiva y emocional a la cual se enfrentan las personas con dicha condición. Es posible que al verse abrumadas por múltiples tareas diarias, opten por priorizar actividades más inmediatas, entre las que no se incluye la toma de los medicamentos antihipertensivos $(23,26)$.

Sin embargo, la información publicada sobre el papel del estrés crónico como mediador de la relación entre la posición socioeconómica y el cumplimiento del tratamiento antihipertensivo farmacológico en 
Latinoamérica, es escasa (27-30). En Colombia, los estudios publicados se han centrado en aclarar la forma en que el estrés predispone a esta condición o a su falta de control (31-35).

En ese orden de ideas, este estudio espera contribuir al conocimiento científico disponible en Colombia y Latinoamérica sobre la relación entre el estrés crónico y el cumplimiento del tratamiento farmacológico antihipertensivo, explorando el papel del primero como mediador entre la posición socioeconómica y el cumplimiento del tratamiento en pacientes hipertensos de 45 a 70 años de tres ciudades de Colombia, en el 2015 y el 2016.

\section{Materiales y métodos}

\section{Muestra}

Los datos de este estudio provienen del programa "De todo corazón" desarrollado por el Grupo de Investigación de Factores Psicosociales en Psicología Clínica y de la Salud de la Universidad de los Andes. Este programa incluyó pacientes de 45 a 70 años de edad vinculados a programas de hipertensión arterial en Bogotá, Medellín y Quibdó. Estas ciudades difieren en términos de niveles de pobreza, oferta de servicios e infraestructura de servicios de salud, especialmente al comparar Quibdó con las otras dos ciudades (36-38).

"De todo corazón" es un programa de carácter longitudinal realizado en dos etapas que utiliza métodos mixtos. En la primera etapa, se seleccionaron las tres ciudades (Bogotá, Medellín y Quibdó) mediante muestreo de criterio o propósito de tipo heterogéneo, con el fin de examinar las variaciones o similitudes del fenómeno en diferentes contextos (39). En la segunda etapa, se seleccionaron dos entidades administradoras de planes de beneficio en cada ciudad: una con mayor cobertura en el régimen contributivo y, otra, en el subsidiado. Otros criterios preliminares para la selección de estas entidades fueron el contar con programas de hipertensión arterial, tener una cobertura igual o superior al tamaño de la muestra en cada ciudad, poseer un registro actualizado de las personas que formaban parte del programa y la disposición de participar en el estudio.

Por último, los participantes se seleccionaron por muestreo probabilístico estratificado basado en la distribución de edad y sexo, lo que permitió tener una muestra representativa de individuos dentro de cada entidad administradora de planes de beneficio con el mínimo sesgo de selección.

Los criterios de exclusión para los participantes fueron ser pacientes con diagnóstico concomitante de diabetes mellitus o con alguna discapacidad cognitiva, neurológica, motora o psiquiátrica, que les impidiera la adecuada resolución de las pruebas psicológicas, así como no hablar español.

En el estudio se tomó como muestra la primera ola del programa, conformada por 258 pacientes reclutados entre el 2015 y el 2016. En los análisis que se describen a continuación, se usaron los pesos muestrales desarrollados para la primera ola para incorporar elementos como la falta de respuesta y garantizar que, dentro del rango de edad del estudio, la muestra reflejara las características de edad y sexo de la población colombiana.

El programa "De todo corazón" fue aprobado por el Comité de Ética de la Universidad de los Andes mediante el acta 531 del 2015, y el presente estudio fue aprobado y categorizado como estudio sin riesgo por el Comité de Ética de la Escuela de Gobierno Alberto Lleras Camargo de la misma universidad. 


\section{Instrumentos}

La información sobre las variables de interés se obtuvo mediante cuestionarios estandarizados. Para conocer las dimensiones de tales cuestionarios, se hicieron análisis factoriales con los pesos muestrales estimados por el programa (40).

\section{Cumplimiento del tratamiento farmacológico}

Se usó la escala de cumplimiento del tratamiento farmacológico de Morisky (41), la cual fue creada para medir el cumplimiento del tratamiento farmacológico en pacientes hipertensos mediante la respuesta a cuatro preguntas dicotómicas sobre la toma de medicamentos por parte de los pacientes y las circunstancias en las que dejan de tomarlos (42). Esta escala se ha validado en Brasil, Perú y España, países de habla española o con contextos similares al de Colombia $(43,44)$. En este estudio, el alfa de Cronbach para dicha escala fue 0,54 , por lo cual se decidió crear un índice a partir de las preguntas, como se ha hecho en otros estudios $(8,10)$.

Las preguntas de la escala son las siguientes: 1) ¿Alguna vez olvida tomar la medicación para la hipertensión arterial? 2) ¿Algunas veces olvida tomar los medicamentos a las horas indicadas? 3) Cuando se siente mejor, ¿a veces deja de tomar la medicación? y, por último, 4) Cuando se siente mal ¿a veces deja de tomar la medicación? Las opciones de respuesta eran "si" o "no", y se asignaba un punto a cada respuesta afirmativa y cero a cada respuesta negativa. Se consideró como pacientes que cumplían el tratamiento a aquellos que respondieron negativamente a todas las preguntas, y que lo incumplían, a aquellos que respondieron a una o más preguntas afirmativamente.

\section{Estrés crónico}

Para medir el estrés crónico se usó la escala de Cohen, et al. (45), con la cual se evalúa el nivel de estrés percibido por las propias personas durante el último mes. Consta de 14 ítems cuyas respuestas se califican en una escala de cinco puntos (45). En este estudio, se obtuvo un alfa de Cronbach de 0,85 , lo que se considera como indicativo de buena fiabilidad. Además, en el análisis factorial se determinó un único factor con un valor propio (Eigenvalue) de 6,4 que acumuló el $45 \%$ de la varianza de la escala. Este mismo cuestionario se validó en Colombia con un alfa de 0,87 y el análisis factorial mostró dos factores (afrontamiento y percepción del estrés) que explicaron el $49,6 \%$ de la varianza (46). Además, se ha encontrado que la escala de Cohen tiene una relación significativa con los niveles de cortisol en el cabello (47), el cual es un biomarcador que ha demostrado ser confiable para medir el estrés crónico (48-50).

\section{Posición socioeconómica}

Para las variables de posición socioeconómica, se usó la versión larga del cuestionario "Nivel socioeconómico en la vida adulta" propuesto por la Red MacArthur en el 2009 para medir el estatus socioeconómico y la salud, el cual consta de 12 preguntas (51). De él se tomaron las variables de nivel educativo y posición en la comunidad, la primera de estas categorizada como "sin primaria, primaria, bachillerato y más de bachillerato", en tanto que la variable de posición en la comunidad se usó de forma continua. 
Para establecer la variable de posición en la comunidad, se les indicaba a los participantes que seleccionaran en una escalera de diez peldaños aquel en el que consideraban que se encontraban, correspondiendo los más altos a una posición más alta en la comunidad y los más bajos a una más baja.

\section{Covariables}

La información sobre las covariables también se obtuvo utilizando cuestionarios estandarizados. Para la variable de apoyo social, se utilizó el cuestionario Medical Outcomes Study-Social Support Survey (MOSSSS), escala de Sherbourne, et al. (52) ya validada en Colombia (53). Este cuestionario consta de 20 ítems y evalúa cuatro componentes: apoyo emocional e informativo, apoyo instrumental, interacción social positiva y apoyo afectivo, y puede usarse como una escala global que reúne todos estos componentes. En el análisis factorial, se encontró que el primer componente tenía un valor propio de 12,8 y acumulaba el $68 \%$ de la varianza, con un alfa de 0,96. Para efectos de este estudio, se usó la escala de forma global.

Otras covariables del estudio fueron la etnicidad asignada por observación (minorías y no minorías), el sexo (hombres y mujeres), la edad (medida de manera continua en años cumplidos) y la ciudad de residencia del participante (Bogotá, Quibdó o Medellín).

\section{Análisis estadístico}

Inicialmente, se hizo un análisis descriptivo seguido de uno bivariado mediante regresiones simples entre las variables de posición socioeconómica y estrés crónico, lo que permitió conocer la distribución y la asociación del estrés crónico con las variables de posición socioeconómica. En segundo lugar, se utilizaron funciones logit multivariadas para evaluar el efecto mediador del estrés crónico entre la posición socioeconómica y el cumplimiento del tratamiento farmacológico, teniendo en cuenta los criterios de mediación propuestos anteriormente $(54,55)$.

Freedman y Schatzkin propusieron dos pasos para explorar la mediación mediante regresiones (56): 1) usar un modelo con el predictor $(X)$ y la variable de resultado $(Y): X->$, y 2) usar un segundo modelo con la variable predictora, la de mediación $(\mathrm{M})$ y la de resultado: $\mathrm{X}->\mathrm{M}->\mathrm{Y}$ para, posteriormente, comparar el coeficiente de $X$ en ambos modelos, esperando encontrar una disminución de la significación estadística y del efecto entre el predictor y la variable de resultado, esto en caso de presentarse una mediación. Los dos modelos se ajustaron con las covariables descritas previamente.

Para la selección de los modelos, se utilizaron los criterios de información bayesianos y de Akaike; asimismo, se estimó el factor de inflación de la varianza y, por último, se usaron errores estándar consistentes.

Los niveles de significación se determinaron a partir de un valor de $p$ menor o igual a 0,1 dadas las características de medición de las variables de interés y el tamaño de la muestra (57). Todos los análisis se hicieron con el programa Stata $14^{\mathrm{TM}}$.

\section{Resultados}

La muestra estudiada incluyó 258 pacientes hipertensos, 161 mujeres y 97 hombres, de los cuales el $33 \%$ residía en Bogotá, el $37 \%$ en Medellín y el $30 \%$ en Quibdó. El $39 \%$ de los pacientes pertenecía a minorías étnicas. 
En cuanto al cumplimiento del tratamiento, el $73 \%$ no lo cumplía; más del 60 $\%$ de los participantes no tenía estudios universitarios y el $48 \%$ no estaba empleado. La media de edad fue de 58 años, con un promedio de 16,62 y 78,37 para las escalas de estrés crónico y apoyo social, respectivamente. La variable de posición en la comunidad tuvo una media de 5,097 (cuadro 1).

\section{Regresiones simples}

Distribución del estrés según las variables de posición socioeconómica. En cuanto a la distribución y asociación del estrés con la posición socioeconómica (cuadro 2), se encontró que las personas con mayores niveles educativos, es decir, bachillerato y por encima de bachillerato, tuvieron, respectivamente, puntajes promedios de estrés crónico 2,98 y 2,49 mayores que las personas con menor nivel educativo (sin primaria). Por otro lado, por cada peldaño adicional de posición social ocupado en la comunidad, las personas disminuyeron, en promedio, 0,42 puntos en la escala de estrés $(p=0,06)$.

Los modelos multivariados finales (cuadro 3) se eligieron teniendo en cuenta los criterios de bondad de ajuste tradicionales: AIC (Akaike Information Criterion) y BIC (Bayesian Information Criterion), y la prueba de multicolinealidad fue menor de 10.

Modelo 1: posición socioeconómica y cumplimiento del tratamiento farmacológico. Las personas con menor nivel de educación reportaron menores probabilidades de cumplir el tratamiento. Por ejemplo, la probabilidad de cumplir el tratamiento de aquellos con educación primaria aumentó 0,35 veces en comparación con las personas sin primaria, en tanto que quienes tenían un nivel superior al bachillerato la aumentaron en 0,18 veces; ambas categorías de educación tuvieron un valor de $p>0,1$. En contraste, en promedio, por cada peldaño de más en la posición en su comunidad, su probabilidad de cumplir el tratamiento disminuyó 0,76 veces $(p=0,2)$.

Cuadro 1. Datos descriptivos de los pacientes hipertensos participantes de Bogotá, Medellín y Quibdó

\begin{tabular}{lrc}
\hline Variables & $\mathbf{n}$ & Media o porcentaje (\%) \\
\hline Cumplimiento del tratamiento & $\mathbf{2 5 0}$ & \\
No & 182 & 73 \\
Estrés & 258 & 16,62 (DE=8,75; rango: 0 a 42) \\
Educación & 258 & \\
Sin primaria & 49 & 19 \\
Primaria & 59 & 23 \\
Bachillerato & 57 & 22 \\
Más que bachillerato & 93 & 36 \\
Posición en la comunidad & 257 & 5,09 (DE=2,40; rango: 1 a 10) \\
Sexo & 258 & \\
Hombre & 98 & 38 \\
Edad & 258 & 58 (DE=6,46; rango: 5 a 70) \\
Etnicidad & 258 & \\
No perteneciente a minorías & 157 & 61 \\
Minorías & 101 & 39 \\
Ciudad & 258 & \\
Bogotá & 85 & 33 \\
Medellín & 96 & 30 \\
Quibdó & 77 & 37 \\
Apoyo social & 258 & 78,37 (DE=17,77; rango: 0 a 95) \\
\hline
\end{tabular}

Nota: en la columna de "media o porcentaje (\%)" se presentan en paréntesis las desviaciones estándar (DE) y los rangos de las variables continuas. 
Modelo 2: posición socioeconómica, estrés crónico y cumplimiento del tratamiento farmacológico. La variable de educación mantuvo la misma tendencia encontrada en el modelo 1, es decir, las personas con mayor nivel educativo tenían más probabilidades de cumplir el tratamiento que aquellas sin primaria, con un valor de $p$ mayor de 0,1 . En cuanto a la variable de posición en la comunidad, se encontró que, en promedio, por cada peldaño de más en la comunidad la probabilidad de cumplir el tratamiento disminuyó 0,11 veces $(p=0,06)$, lo que denota un aumento en la significación estadística y el coeficiente. En cuanto al estrés y el cumplimiento, se estableció una asociación significativa y negativa $(p=0,001)$, lo que indica que las personas con mayores niveles de estrés crónico eran las que menos cumplían el tratamiento.

Es preciso indicar que la relación entre la variable de nivel educativo y el cumplimiento del tratamiento no presentó cambios en su significación estadística al incluir la variable de estrés; sin embargo, cuando se revisó la relación entre la posición en la comunidad y el cumplimiento del tratamiento

Cuadro 2. Distribución del estrés en función de las variables de posición socioeconómica mediante regresiones lineales simples

\begin{tabular}{|c|c|c|}
\hline $\begin{array}{l}\text { Variables de posición } \\
\text { socioeconómica }\end{array}$ & Coeficiente B & EE \\
\hline \multicolumn{3}{|c|}{$\begin{array}{l}\text { Educación (categoría de referencia } \\
\text { "Sin primaria") }\end{array}$} \\
\hline Primaria & 1,61 & 1,69 \\
\hline Bachillerato & $2,98^{*}$ & 1,70 \\
\hline Más de bachillerato & $2,49^{*}$ & 1,55 \\
\hline Posición en la comunidad & $-0,42^{*}$ & 0,22 \\
\hline \multicolumn{3}{|l|}{ EE: error estándar } \\
\hline \multicolumn{3}{|c|}{$\begin{array}{l}\text { La variable dependiente de los modelos fue la escala de estrés. Estos modelos } \\
\text { se utilizaron sin pesos muestrales. La variable de posición en la comunidad } \\
\text { se midió en una escalera de uno a diez peldaños, en la que los más altos } \\
\text { correspondían a una mejor posición en la comunidad. }\end{array}$} \\
\hline
\end{tabular}

Cuadro 3. Resultado del modelo de ecuaciones estructurales para las variables

\begin{tabular}{lcccc}
\hline Variables (variable de referencia) & \multicolumn{2}{c}{$\begin{array}{c}\text { Modelo 1 } \\
\text { (Cumplimiento) }\end{array}$} & $\begin{array}{c}\text { Modelo 2 } \\
\text { (Cumplimiento) }\end{array}$ \\
\cline { 2 - 5 } & (OR) & (EE) & OR & (EE) \\
\hline Estrés & & & $-0,05^{\star \star *}$ & 0,01 \\
Educación (Sin primaria) & 0,35 & 0,35 & 0,48 & 0,38 \\
$\quad$ Primaria & 0,11 & 0,41 & 0,33 & 0,44 \\
$\quad$ Bachillerato & 0,18 & 0,38 & 0,40 & 0,42 \\
$\quad$ Más que bachillerato & $-0,07$ & 0,06 & $-0,11^{\star}$ & 0,06 \\
$\quad$ Posición en la comunidad & & & & \\
Sexo (Mujer) & $-0,21$ & 0,26 & $-0,30$ & 0,25 \\
$\quad$ Hombre & 0,02 & 0,01 & $-0,20$ & 0,01 \\
$\quad$ Edad & & & & \\
Etnicidad (No perteneciente a minorías) & 0,24 & 0,37 & 0,29 & 0,35 \\
$\quad$ Minorías & & & & \\
Ciudad (Bogotá) & & & & \\
$\quad$ Medellín & $-0,49^{*}$ & 0,30 & $-0,56^{*}$ & 0,31 \\
$\quad$ Quibdó & $-1,02^{\star *}$ & 0,45 & $-1,08^{\star *}$ & 0,45 \\
$\quad$ Apoyo social & 0,003 & 0,007 & $-0,01$ & 0,007 \\
\hline
\end{tabular}

OR: odds ratio; EE: error estándar

${ }^{*} p \leq 0,1 ;{ }^{* *} p \leq 0,05 ;{ }^{* * *} p \leq 0,01$

Los modelos 1 y 2 tienen como variable de resultado el cumplimiento. El modelo 1 no incluyó la variable estrés. Los dos modelos se ajustaron por sexo, edad, etnicidad, ciudad y apoyo social. 
en presencia de la variable estrés, se encontró que la significación estadística y el efecto aumentaron, por lo que no se cumplió el supuesto de mediación planteado por Freedman y Schatzkin (56).

Por último, tanto en el modelo 1 como en el 2, las personas que vivían en Quibdó y Medellín tenían menores probabilidades de cumplir con el tratamiento $(p \leq 0,1)$ en comparación con quienes vivían en Bogotá.

\section{Discusión}

En este estudio se examinó el papel del estrés crónico como mediador de la relación entre la posición socioeconómica y el cumplimiento del tratamiento farmacológico en pacientes hipertensos de tres ciudades de Colombia. Hasta donde se pudo constatar, este es el primero de este tipo que se realiza en Colombia. Los principales hallazgos sugieren que el estrés no es un mediador entre la posición socioeconómica y el cumplimiento del tratamiento. Se establecieron asociaciones significativas entre las diferentes variables de interés y la asociación mencionada aumentó al incluir la variable del estrés. Además, hubo algunos hallazgos novedosos que se discuten a continuación.

Se encontró que los participantes con mayor nivel educativo reportaban mayores niveles de estrés, lo que en algunos casos se contrapone y en otros coincide con algunos estudios $(58,59)$ : en una muestra de 283 hombres, Landsbergis, et al., establecieron que las personas más educadas presentaban menores niveles de estrés (58), en tanto que, en una muestra de 444 mujeres, Garay, et al. reportaron que, a mayor educación, mayores los niveles de estrés (59), lo que, según los autores, se debe a que muchas mujeres mayores de 40 años en Latinoamérica aún cumplen una doble función social, pues su mayor nivel educativo aumenta sus posibilidades de incorporarse a la fuerza laboral, y ello se suma a las responsabilidades del hogar que muchas de ellas todavía cumplen (59). Esta situación podría aumentar la carga cognitiva derivada de las múltiples actividades que deben realizar.

El argumento expuesto por Garay, et al., podría explicar el por qué en el presente estudio los participantes más educados presentaron mayores niveles de estrés, ya que, de los 258 participantes, 161 eran mujeres con una media de edad de 58 años.

En cuanto a la posición socioeconómica subjetiva o posición en la comunidad y el estrés, se determinó que, por cada peldaño adicional en su posición en la comunidad, las personas reportaban menores niveles de estrés crónico, lo que va en la misma línea de lo presentado por Tang, et al., en un metaanálisis (60) en el cual concluyeron que las personas que se percibían en una mejor posición en la escala social reportaban mejores resultados en salud.

Por otro lado, aunque los resultados de los dos modelos empleados difirieron en la significación y la magnitud de los coeficientes, las tendencias fueron las mismas en ambos. El resultado derivado de los dos modelos en cuanto a la relación entre la posición socioeconómica subjetiva y el cumplimiento del tratamiento farmacológico contrasta con lo hallado en otros estudios (61), ya que, por cada peldaño en la escalera de posición en la comunidad, las personas tuvieron menores probabilidades de cumplir con el tratamiento, en tanto que, en su estudio, Demakakos, et al. hallaron que, a mayor posición socioeconómica subjetiva, mejores resultados cardiovasculares (61). Por su parte, Tang, et al. refieren que el instrumento de MacArthur usado para medir dicha posición podría presentar un sesgo de 
respuesta (60). Por ejemplo, las personas que se clasifican en lo alto de la escalera podrían verse afectadas por el fenómeno de la deseabilidad social, lo que explicaría que quienes menos cumplen con el tratamiento reporten una posición socioeconómica subjetiva alta.

Ahora bien, la razón de que la variable de la posición en la comunidad esté asociada negativamente con la variable de cumplimiento del tratamiento, sería que, cuanto mejor es la posición en la comunidad, mayor es la demanda por elevarla, lo que implicaría tener menos tiempo para tomar los medicamentos y una mayor carga cognitiva, con el consecuente incumplimiento del tratamiento. Las personas con liderazgo en sus comunidades suelen reportar puntajes altos en la escala de posición socioeconómica subjetiva y mayores niveles de estrés $(52,62)$. Por ello, es factible que dicha condición pueda interferir, en alguna medida, con el cumplimiento del tratamiento.

Además de lo ya mencionando, el hecho de tener un nivel educativo más alto resultó en un mejor cumplimiento del tratamiento, resultado similar al del estudio de Chow, et al. (11).

En el presente estudio, la relación entre el estrés crónico y el cumplimiento del tratamiento farmacológico fue negativa, pues los puntajes más altos en la escala de estrés se relacionaron con una menor probabilidad de cumplimiento. Este hallazgo es consistente con lo encontrado por Marshall, et al. en una revisión sistemática de estudios en 19 países, en la cual el estrés afectó negativamente dicho cumplimiento (23).

En cuanto al hecho de que a mayores niveles de estrés, menor es la probabilidad de cumplir con el tratamiento, diversos autores han explicado que ello puede deberse a que los pacientes con síntomas de estrés serían más propensos a los efectos negativos de los medicamentos dada su condición de irritabilidad o ansiedad $(23,63)$. Otra posible explicación es que las personas con mayores niveles de estrés crónico sean quienes tienen una mayor carga cognitiva, lo que les dificulta priorizar actividades como la toma de los medicamentos en las horas y las cantidades indicadas.

Un hallazgo coincidente en el presente estudio es que el cumplimiento difirió según el lugar de residencia, pues en Quibdó, la ciudad con el menor desarrollo socioeconómico, la probabilidad de cumplir con el tratamiento fue 1,02 veces menor que en Bogotá. Aunque no se encontraron otros estudios que compararan diversas zonas urbanas (64), en el país, sí se han reportado diferencias entre lo urbano y lo rural. En su estudio, Camacho, et al., encontraron que las personas residentes en las zonas rurales de Colombia con menor desarrollo socioeconómico cumplían menos con el tratamiento que quienes vivían en zonas urbanas (65).

Algunas limitaciones del presente estudio merecen consideración. Primero, la muestra fue limitada en términos de diversidad racial (una sola observación en población indígena); sin embargo, incluso unificando las observaciones en población indígena y afrocolombiana bajo el criterio de minorías étnicas, no se evidenció una asociación significativa. Segundo, el tamaño de la muestra (258 participantes) limitó el poder estadístico para detectar asociaciones significativas, pero de todas maneras se encontraron relaciones significativas entre las variables de interés. Tercero, la información provenía de los propios participantes, incluida la medición de la variable de estrés en una escala cuyas preguntas tenían como referencia el último mes, lo que podría implicar un 
sesgo de memoria, aunque debe mencionarse que, según diferentes estudios, esta escala tiene similitud con biomarcadores del estrés crónico (47-50).

La valoración indirecta del cumplimiento del tratamiento está relacionada con posibles sesgos de información, como la deseabilidad social. Sin embargo, el cuestionario empleado se creó para valorar el cumplimiento del tratamiento en pacientes con hipertensión arterial y, además, es breve y muy fácil de aplicar, tiene una gran especificidad y un gran valor predictivo positivo, así como poca exigencia para su comprensión, y fue validado en su versión española por Val, et al. (43), y se ha usado previamente en Colombia (10).

Por último, debe mencionarse el sesgo de autoselección. Los pacientes que hicieron parte de esta muestra podrían considerarse como los que más cumplían el tratamiento, dadas su disposición para ser parte de un programa de hipertensión arterial y su voluntad de participar en este estudio. En otras personas, el cumplimiento podría ser menor debido a diversos factores psicosociales y biológicos que interferirían en la toma de sus medicamentos.

A pesar de todas las limitaciones, el estudio sugiere que el estrés crónico no es un mediador entre la posición socioeconómica y el cumplimiento del tratamiento farmacológico en pacientes hipertensos inscritos en programas de hipertensión en Quibdó, Bogotá y Medellín, aunque permite plantear la hipótesis de que puede ser un elemento modificador de dicha relación. Estos hallazgos no son concluyentes, por lo que deben considerarse con cautela, ya que la muestra no fue lo suficientemente amplia para el país y las asociaciones significativas se establecieron a partir de un valor de $p$ de 0,1 o menos.

En futuros estudios en el país, deberá contarse con muestras más amplias y representativas y con mayor participación de minorías étnicas, y medir el cumplimiento y el estrés crónico con otros cuestionarios para indagar sobre el mecanismo moderador del estrés en esta relación. Se sugiere, asimismo, incorporar otros grupos poblacionales pertenecientes a diferentes niveles socioeconómicos que no necesariamente estén en programas de hipertensión arterial.

Por otro lado, los proveedores de servicios de salud deberían evaluar sistemáticamente signos de estrés crónico en sus pacientes como un factor de riesgo para el incumplimiento del tratamiento farmacológico.

\section{Agradecimientos}

Los autores agradecemos al equipo del programa "De todo corazón" por los comentarios hechos al manuscrito.

\section{Referencias}

1. Gasperin D, Netuveli G, Dias-da-Costa JS, Pattussi MP. Effect of psychological stress on blood pressure increase: A meta-analysis of cohort studies. Cad Saúde Pública. 2009;25:715-26. https://doi.org/10.1590/S0102-311X2009000400002

2. Kearney PM, Whelton M, Reynolds K, Muntner P, Whelton PK, He J. Global burden of hypertension: Analysis of worldwide data. Lancet. 2005;365:217-23. https://doi.org/10.1016/S0140-6736(05)17741-1

3. Sarki AM, Nduka CU, Stranges S, Kandala NB, Uthman OA. Prevalence of hypertension in low- and middle-income countries: A systematic review and meta-analysis. Medicine (Baltimore). 2015;94:1-16. https://doi.org/10.1097/MD.0000000000001959

4. World Health Organization. Causes of death 2008: Data sources and methods Fecha de consulta: 29 de junio de 2018. Disponible en: http://www.who.int/healthinfo/global burden disease/cod 2008 sources methods.pdf 
5. Lim SS, Vos T, Flaxman AD, Danaei G, Shibuya K, Adair-Rohani H, et al. A comparative risk assessment of burden of disease and injury attributable to 67 risk factors and risk factor clusters in 21 regions, 1990-2010: A systematic analysis for the Global Burden of Disease Study 2010. Lancet. 2012;380:2224-60. https://doi.org/10.1016/S0140-6736(12)61766-8

6. Lucumí-Cuesta DI. Disparities in hypertension in Colombia: A mixed-method study. Fecha de consulta: 22 de junio de 2018. Disponible en: https://deepblue.lib.umich.edu/bitstream/ handle/2027.42/107278/dilucumi 1.pdf

7. Instituto Nacional de Salud, Observatorio Nacional de Salud. Quinto informe ONS - Carga de enfermedad por enfermedades crónicas no transmisibles y discapacidad en Colombia. Fecha de consulta: 24 de junio de 2018. Disponible en: https://www.minsalud.gov.co/sites/rid/ Lists/BibliotecaDigital/RIDE/IA/INS/informe-ons-5.pdf

8. Castaño-Castrillón JJ, Echeverri-Rubio C, Giraldo-Cardona JF, Maldonado-Mora Á, Melo-Parra J, Meza-Orozco GA, et al. Adherencia al tratamiento de pacientes hipertensos atendidos en Assbasalud ESE, Manizales (Colombia) 2011. Revista de la Facultad de Medicina. 2012;60:179-97.

9. Guarín-Loaiza GM, Pinilla-Roa AE. Adherencia al tratamiento antihipertensivo y su relación con la calidad de vida en pacientes de dos hospitales de Bogotá, D.C. 2013-2014. Revista de la Facultad de Medicina. 2016;64:651-7. https://doi.org/10.15446/revfacmed.v64n4.52217

10. Casas MC, Chavarro LM, Cardona D. Adhesión al tratamiento de la hipertensión arterial en dos municipios de Colombia. 2010-2011. Hacia la Promoción la Salud. 2013;18:81-96.

11. Chow CK, Teo KK, Rangarajan S, Islam S, Gupta R, Avezum A, et al. Prevalence, awareness, treatment, and control of hypertension in rural and urban communities in high-, middle-, and low-income countries. JAMA. 2013;310:959-68. https://doi.org/10.1001/jama.2013.184182

12. Rolnick SJ, Pawloski PA, Hedblom BD, Asche SE, Bruzek RJ. Patient characteristics associated with medication adherence. Clin Med Res. 2013;11:54-65. https://doi.org/10.3121/cmr.2013.1113

13. Grotto I, Huerta M, Sharabi Y. Hypertension and socioeconomic status. Curr Opin Cardiol. 2008;23:335-9. https://doi.org/10.1097/HCO.0b013e3283021c70

14. Link BG, Phelan JC. Social conditions as fundamental causes of disease social conditions as fundamental causes of disease. J Health Soc Behav. 1995;35:80-94. https://doi.org/10.2307/2626958

15. Adler NE, Boyce T, Chesney M, Cohen S, Folkman S, Kahn RL, et al. Socioeconomic status and health. The challenge of the gradient. Am Psychol. 1994;49:15-24. https://doi.org/10.1037/0003-066X.49.1.15

16. Baum A, Garofalo JP, Yali M. Socioeconomic status and chronic stress. Does stress account for SES effects on health? Ann NY Acad Sci. 1999;896:131-44. https://doi.org/10.1111/i.1749-6632.1999.tb08111.x

17. Colhoun HM, Hemingway H, Poulter NR. Socio-economic status and blood pressure: An overview analysis. J Hum Hypertens. 1998;12:91-110.

18. Cha SH, Park HS, Cho HJ. Socioeconomic disparities in prevalence, treatment, and control of hypertension in middle-aged Koreans. J Epidemiol. 2012;22:425-32. https://doi.org/10.2188/jea.JE20110132

19. Ministerio de Educación. Población colombiana con muy bajo nivel educativo, según los resultados del censo. Fecha de consulta: 18 de septiembre de 2017. Disponible en: http://www.mineducacion.gov.co/cvn/1665/fo-article-99519.pdf

20. Departamento Administrativo Nacional de Estadística. Censo general 2005. Fecha de consulta: 5 de septiembre de 2018. Disponible en: https://www.dane.gov.co/index.php/ estadisticas-por-tema/demografia-y-poblacion/censo-general-2005-1

21. Landsbergis PA, Dobson M, Koutsouras G, Schnall P. Job strain and ambulatory blood pressure: A meta-analysis and systematic review. Am J Public Health. 2013;103:61-71. https://doi.org/10.2105/AJPH.2012.301153

22. Liu MY, Li N, Li WA, Khan H. Association between psychosocial stress and hypertension: A systematic review and meta-analysis. Neurol Res. 2017;39:573-80. https://doi.org/10.1080/01616412.2017.1317904

23. Marshall IJ, Wolfe CD, McKevitt C. Lay perspectives on hypertension and drug adherence: Systematic review of qualitative research. BMJ. 2012;345:e3953.

https://doi.org/10.1136/bmj.e3953 
24. Kershaw KN, Lane-Cordova AD, Carnethon MR, Tindle HA, Liu K. Chronic stress and endothelial dysfunction: The Multi-Ethnic Study of Atherosclerosis (MESA). Am J Hypertens. 2017;30:75-80. https://doi.org/10.1093/ajh/hpw103

25. Mena-Díaz FC, Nazar G, Mendoza-Parra S. Antecedentes de adherencia al tratamiento en pacientes hipertensos de un centro de salud chileno. Hacia la Promoción de la Salud. 2018;23:67-78.

26. Zen AL, Zhao S, Whooley MA, Cohen B. Post-traumatic stress disorder is associated with poor health behaviors: Findings from the heart and soul study. Sport Med. 2012;31:194-201. https://doi.org/10.1037/a0025989

27. Sandoval D, Chacón J, Koch E, Romero T, Henríquez Ó, Muñoz R. Influencia de factores psicosociales en la adherencia al tratamiento farmacológico antihipertensivo. Resultados de una cohorte del Programa de Salud Cardiovascular de la Región Metropolitana, Chile. Rev Med Chil. 2014;142:1245-52. https://doi.org/10.4067/S0034-98872014001000003

28. Estela L, Morales R. La adherencia al tratamiento en las enfermedades crónicas. Revista Cubana de Angiología y Cirugía Vascular. 2015;16:175-89.

29. Chacón J, Sandoval D, Muñoz R, Romero T. Evaluación del control de la presión arterial y la adherencia terapéutica en hipertensos seguidos en el Programa de Salud Cardiovascular (PSCV): asociación con características clínicas, socioeconómicas y psicosociales. Rev Chil Cardiol. 2015;34:18-27. https://doi.org/10.4067/S0718-85602015000100002

30. Trujano R, Ávila D, Vega Z, Nava C. Estrés familiar y adherencia terapéutica en pacientes con enfermedades crónicas. Alternativas en Psicología. 2012;16:78-84.

31. Pérez LH, Gutiérrez LA, Vioque J, Torres Y. Relation between overweight, diabetes, stress and hypertension: A case-control study in Yarumal - Antioquia, Colombia. Eur J Epidemiol. 2001;17:275-80.

32. Campo JE, Reyes JJ, Ortiz C, Quintero L, Herrera JA. Niveles de la presión arterial y de estrés psicosocial en estudiantes de la Facultad de Salud, Universidad del Valle, Cali, Colombia, 2003-2004. Colomb Med. 2006;37:21-5.

33. Herrera E. Adherencia al tratamiento en personas con hipertensión arterial. Avances en Enfermería. 2012;30:67-75.

34. Rodríguez-López M, Varela A M, Rincón-Hoyos H, Velasco M, Caicedo D, Méndez F, et al. Prevalencia y factores asociados a la adherencia al tratamiento no farmacológico en pacientes con hipertensión y diabetes en servicios de baja complejidad. Revista Facultad Nacional de Salud Pública 2015;33:192-9. https://doi.org/10.17533/udea.rfnsp.v33n2a06

35. Legido-Quigley H, Camacho-López PA, Balabanova D, Perel P, López-Jaramillo P, Nieuwlaat $\mathrm{R}$, et al. Patients' knowledge, attitudes, behaviour and health care experiences on the prevention, detection, management and control of hypertension in Colombia: A qualitative study. PLoS One. 2015;10:e0122112. https://doi.org/10.1371/journal.pone.0122112

36. Cortés D, Vargas JF. Inequidad regional en Colombia. Fecha de consulta: 18 de septiembre de 2017. Disponible en: http://repository.urosario.edu.co/bitstream/handle/10336/10980/10081.pdf

37. Ministerio de Salud y Protección Social. Análisis de la Situación de Salud (ASIS) Colombia 2015. Fecha de consulta: 5 de septiembre de 2017. Disponible en: https://www.minsalud.gov. co/sites/rid/Lists/BibliotecaDigital/RIDE/VS/ED/PSP/asis-2015.pdf

38. Secretaría de Salud del Chocó. Análisis de situación de salud (ASIS) con el modelo de los determinantes sociales de salud. Fecha de consulta: 5 de septiembre de 2017. Disponible en: https://www.minsalud.gov.co/sites/rid/Paginas/freesearchresults.aspx?k=\&k=asis\%20 choco $\% 202015$

39. Patton MQ. Qualitative evaluation and research methods. 2nd edition. Thousand Oaks, CA: Sage Publications; 1990. p. 277-82.

40. Acock A. A gentle introduction to Stata. 3rd edition. Texas: Stata Press; 2012.

41. Morisky DE, Green LW, Levine DM. Concurrent and predictive validity of a self-reported measure of medication adherence. Med Care. 1986;24:67-74.

42. Pérez-Escamilla B, Franco-Trigo L, Moullin J, Martínez-Martínez F, García-Corpas J. Identification of validated questionnaires to measure adherence to pharmacological antihypertensive treatments. Patient Prefer Adherence. 2015;9:569-78.

https://doi.org/10.2147/PPA.S76139 
43. Val A, Amorós G, Martínez P, Fernández ML, León M. Descriptive study of patient compliance in pharmacologic antihypertensive treatment and validation of the Morisky and Green test. Atención Primaria.1992;10:767-70.

44. Bastos-Barbosa RG, Ferriolli E, Moriguti JC, Nogueira CB, Nobre F, Ueta J, et al. Treatment adherence and blood pressure control in older individuals with hypertension. Arq Bras Cardiol. 2012;99:636-41. https://doi.org/10.1590/S0066-782X2012005000054

45. Cohen S, Kamarck T, Mermelstein R. A global measure of perceived stress. J Health Soc Behav. 1983;24:385-96.

46. Campo-Arias A, Bustos-Leiton G, Romero-Chaparro A. Consistencia interna y dimensionalidad de la Escala de Estrés Percibido (EEP-10 y EEP-14) en una muestra de universitarias de Bogotá, Colombia. Aquichan. 2009;9:271-80.

47. van Uum SH, Sauvé B, Fraser LA, Morley-Forster P, Paul TL, Koren G. Elevated content of cortisol in hair of patients with severe chronic pain: A novel biomarker for stress. Stress. 2008;11:483-8. https://doi.org/10.1080/10253890801887388

48. Glei DA, Goldman N, Shkolnikov VM, Jdanov D, Shkolnikova M, Vaupel JW, et al. Perceived stress and biological risk: Is the link stronger in Russians than in Taiwanese and Americans? Stress. 2014;16:411-20. https://doi.org/10.3109/10253890.2013.789015

49. Kalra S, Einarson A, Karaskov T, Uum Sv, Koren G. The relationship between stress and hair cortisol in healthy pregnant women. Clin Invest Med. 2007;30:E100-4. https://doi.org/10.25011/cim.v30i2.986

50. O'Brien KM, Tronick EZ, Moore CL. Relationship between hair cortisol and perceived chronic stress in a diverse sample. Stress Heal. 2013;29:337-44. https://doi.org/10.1002/smi.2475

51. Adler N, Stewart J. The MacArthur Scale of Subjective Social Status. Fecha de consulta: 5 de septiembre de 2017. Disponible en: https://macses.ucsf.edu/research/psychosocial/ subjective.php

52. Sherbourne CD, Stewart AL. The MOS social support survey. Soc Sci Med. 1991;32:705-14.

53. Londoño NH, Rogers HL, Castilla JF, Posada SL, Ochoa NL, Jaramillo MA, et al. Validación en Colombia del cuestionario MOS de apoyo social. Int J Psychol Res. 2012;5:142-50.

54. Fritz MS, MacKinnon DP. A graphical representation of the mediated effect. Behav Res Methods. 2008;40:55-60. https://doi.org/10.3758/BRM.40.1.55

55. MacKinnon DP, Lockwood CM, Hoffman JM, West SG, Sheets V. A comparison of methods to test mediation and other intervening variable effects. Psychol Methods. 2002;7:83-104. https://doi.org/10.1037/1082-989X.7.1.83

56. Freedman LS, Schatzkin A. Sample size for studying intermediate endpoints within intervention trials of observational studies. Am J Epidemiol. 1992;136:1148-59.

57. Wasserstein RL, Lazar NA. The ASA's statement on $p$-values: Context, process, and purpose. Am Stat. 2016;70:129-33. https://doi.org/10.1080/00031305.2016.1154108

58. Landsbergis PA, Schnall PL, Pickering TG, Warren K, Schwartz JE. Lower socioeconomic status among men in relation to the association between job strain and blood pressure. Scand J Work Environ Health. 2003;29:206-15. https://doi.org/10.5271/sjweh.723

59. Garay J, Farfán M. Niveles de depresión, autoestima y estrés en mujeres que tienen un trabajo remunerado y mujeres que tienen un trabajo no remunerado (amas de casa). Psicología Iberoamericana. 2007;15:22-9.

60. Tang KL, Rashid R, Godley J, Ghali WA. Association between subjective social status and cardiovascular disease and cardiovascular risk factors: A systematic review and metaanalysis. BMJ Open. 2016;6:e010137. https://doi.org/10.1136/bmjopen-2015-010137

61. Demakakos P, Nazroo J, Breeze E, Marmot M. Socioeconomic status and health: The role of subjective social status. Soc Sci Med. 2008;67:330-40. https://doi.org/10.1016/j.socscimed.2008.03.038

62. Baysak B, Yener MI. The relationship between perceived leadership style and perceived stress on hospital employees. Procedia - Soc Behav Sci. 2015;207:79-89. https://doi.org/10.1016/j.sbspro.2015.10.159

63. Kretchy IA, Owusu-Daaku FT, Danquah S. Locus of control and anti-hypertensive medication adherence in Ghana. Pan Afr Med J. 2014;17:1-13. 
64. Isaza C.A, Moncada J.C, Mesa G, Osorio FJ. Efectividad del tratamiento antihipertensivo en una muestra de pacientes colombianos. Biomédica. 2004;24:273-81.

https://doi.org/10.7705/biomedica.v24i3.1273

65. Camacho PA, Gómez-Arbeláez D, Molina DI, Sánchez G, Arcos E, Narváez C, et al. Social disparities explain differences in hypertension prevalence, detection and control in Colombia. J Hypertens. 2016;34:2344-52. https://doi.org/10.1097/HJH.0000000000001115 